\title{
On strongly monotone flows
}

\author{
by WolfGang Walter (Karlsruhe)
}

Wtodzimierz Mlak in memoriam

\begin{abstract}
M. Hirsch's famous theorem on strongly monotone flows generated by autonomous systems $u^{\prime}(t)=f(u(t))$ is generalized to the case where $f$ depends also on $t$, satisfies Carathéodory hypotheses and is only locally Lipschitz continuous in $u$. The main result is a corresponding Comparison Theorem, where $f(t, u)$ is quasimonotone increasing in $u$; it describes precisely for which components equality or strict inequality holds.
\end{abstract}

1. Introduction. One of M. Hirsch's theorems on monotone flows [1] states that the flow generated by a $C^{1}$-function $f$ is strongly monotone if the Jacobian $f^{\prime}(x)=\left(\partial f_{i} / \partial x_{j}\right)$ is essentially positive (i.e., $\partial f_{i} / \partial x_{j} \geq 0$ for $i \neq j$ ) and irreducible. In the language of differential equations the theorem says that the conditions

$$
v^{\prime}=f(v), \quad w^{\prime}=f(w) \quad \text { in } J=[a, b], \quad v(a) \leq w(a), \quad v(a) \neq w(a)
$$

imply the strict inequality $v(t)<w(t)$ in $J_{0}=(a, b]$. Here, $f: \mathbb{R}^{n} \supset D \rightarrow \mathbb{R}^{n}$ and $v, w: J \rightarrow \mathbb{R}^{n}$, while $\leq$ and $<$ refer to the componentwise ordering in $\mathbb{R}^{n}$.

Hirsch's original proof was subject to criticism, and other proofs have been given by several authors. Our objective is to present a simple proof for a more general theorem. We allow that $f$ depends explicitly on $t$ and satisfies only Carathéodory hypotheses. Furthermore, we consider the case where $v$, $w$ are not necessarily solutions but satisfy differential inequalities, and finally we assume that $f(t, x)$ is only locally Lipschitzian in $x$; let us remark that there are important applications, e.g., in nonsymmetric mechanical systems, where $f$ is Lipschitzian but not of class $C^{1}$. Consequently, the handsome irreducibility assumption has to be replaced by an assumption that avoids derivatives.

1991 Mathematics Subject Classification: 34A40, 34C11, 34C35.

Key words and phrases: system of ordinary differential equations, initial value problem, comparison theorem, monotone flow, quasimonotonicity. 
Theorem 1 deals with a system of linear differential inequalities. The main point in this theorem is the assertion that each component of $u(t)$ is either strictly positive in $(a, b]$ or vanishes in an interval $\left[a, a+\delta_{i}\right]$ and is positive thereafter. Theorem 2 , which covers the nonlinear case, is reduced to Theorem 1 by simple, well-known means. From these results, rather weak additional assumptions which imply strict inequalities in $J_{0}$ for all components are easily obtained.

2. The linear case. For $x, y \in \mathbb{R}^{n}$ we define

$$
x \leq y \Leftrightarrow x_{i} \leq y_{i} \quad \text { for } i \in N \quad \text { and } \quad x<y \Leftrightarrow x_{i}<y_{i} \quad \text { for } i \in N \text {, }
$$

where $N=\{1, \ldots, n\}$. The spaces $A C(J)$ and $L(J)$ contain all functions $x(t)$ that are absolutely continuous or integrable in $J$, resp. Here and below, $J=[a, b], J_{0}=(a, b]$ and $e_{n}=(1, \ldots, 1) \in \mathbb{R}^{n}$.

Theorem 1. Let $C(t)=\left(c_{i j}(t)\right) \in L(J)$ be an essentially positive $n \times n$ matrix, i.e., $c_{i j} \geq 0$ a.e. in $J$ for $i \neq j$. Then $u \in A C(J)$,

$$
u(a) \geq 0, \quad u^{\prime} \geq C u \quad \text { a.e. in } J
$$

imply $u(t) \geq 0$ in J. Moreover, the index set $N$ can be split up in two disjoint sets $\alpha, \beta(\alpha \cup \beta=N, \alpha \cap \beta=\emptyset)$ such that

- for $i \in \alpha, u_{i}(t)>0$ in $J_{0}$,

- for $j \in \beta, u_{j}(t)=0$ in $\left[a, a+\delta_{j}\right]$ and $u_{j}(t)>0$ in $\left(a+\delta_{j}, b\right]$,

where $\delta_{j}>0$.

Proof. Let $\left|c_{i j}(t)\right| \leq m(t) \in L(J)$ for $i, j \in N$ and $M(t)=\int_{a}^{t} m(s) d s$. The function $w(t)=e^{M(t)} u(t)$ satisfies $w(a) \geq 0$ and

$$
w^{\prime} \geq D(t) w \quad \text { a.e. in } J, \quad \text { where } D(t)=C(t)+m(t) I \geq 0,
$$

i.e., $d_{i j}(t) \geq 0$ for all $i, j \in N$. The function

$$
h(t)=(\varrho, \ldots, \varrho) \quad \text { with } \varrho(t)=e^{(n+1) M(t)}
$$

satisfies $h(a)=e_{n}=(1, \ldots, 1)$ and $h^{\prime} \geq D h$. Hence $w_{\varepsilon}=w+\varepsilon h(\varepsilon>0)$ has the properties

$$
w_{\varepsilon}^{\prime} \geq D w_{\varepsilon} \quad \text { a.e. in } J \text { and } w(a) \geq \varepsilon e_{n}>0 .
$$

As long as $w_{\varepsilon} \geq 0$, we have $w_{\varepsilon}^{\prime} \geq 0$. It follows easily that $w_{\varepsilon}(t) \geq \varepsilon e_{n}$ for all $t \in J$. Since $\varepsilon>0$ is arbitrary, $w(t) \geq 0$ in $J$ and also $w^{\prime}(t) \geq 0$ a.e. in $J$.

This shows that $u(t) \geq 0$ in $J$. Assume now that a component $u_{i}$ is positive at $t_{0} \in J$. Then $w_{i}\left(t_{0}\right)>0$ and therefore $w_{i}(t)>0$ for $t>t_{0}$, which in turn implies $u_{i}(t)>0$ for $t>t_{0}$. We let $\alpha$ be the set of all indices $i$ such that $u_{i}>0$ in $J_{0}=(a, b]$. Then each $u_{j}$ with $j \notin \alpha$ vanishes at some point $t_{i} \in J_{0}$ and therefore in $\left[a, t_{i}\right]$. 
3. The nonlinear, quasimonotone case. We consider the nonlinear equation

$$
u^{\prime}(t)=f(t, u(t)) \quad \text { a.e. in } J
$$

and assume for simplicity that $f(t, x)$ is defined in the strip $S=J \times \mathbb{R}^{n}$ and satisfies the following conditions: $f(t, \cdot)$ is continuous in $\mathbb{R}^{n}$ for almost all (fixed) $t \in J, f(\cdot, x)$ is measurable in $J$ for all (fixed) $x \in \mathbb{R}^{n}, f(t, 0) \in L(\mathbb{R})$, and for each constant $A>0$ there is a function $m(t) \in L(J)$ such that

$$
|f(t, x)-f(t, y)| \leq m(t)|x-y| \quad \text { for } t \in J \text { and }|x|,|y| \in A \text {. }
$$

The defect $P$ of a function $v \in A C(J)$ with respect to equation (2) is defined by

$$
(P v)(t)=v^{\prime}(t)-f(t, v(t)) .
$$

The function $f$ is said to be quasimonotone increasing in $x$ if $f_{i}(t, x)$ is (weakly) increasing in $x_{j}$ for all $j \neq i$, or equivalently, if

$$
x \leq y, x_{i}=y_{i} \Rightarrow f_{i}(t, x) \leq f_{i}(t, y) \quad \text { a.e. in } J \quad(i=1, \ldots, n) .
$$

TheOREM 2. Assume that the function $f(t, x)$ satisfies the conditions given above and is quasimonotone increasing in $x$, and let $v, w \in A C(J)$ satisfy

$$
v(a) \leq w(a) \text { and } P v \leq P w \text { a.e. in } J .
$$

Then $v \leq w$ in $J$, and there exist disjoint index sets $\alpha, \beta$ with $\alpha \cup \beta=N$ and positive numbers $\delta_{j}$ such that

$$
v_{i}<w_{i} \quad \text { in } J_{0} \quad \text { for } i \in \alpha,
$$

$$
v_{j}=w_{j} \quad \text { in }\left[a, a+\delta_{j}\right] \quad \text { and } \quad v_{j}<w_{j} \quad \text { in }\left(a+\delta_{j}, b\right] \quad \text { for } j \in \beta \text {. }
$$

Proof. Let $|v(t)|,|w(t)| \leq A$ in $J$ and assume that (3) holds. Let $u(t)=$ $w(t)-v(t)$. In the scalar case $(n=1)$ one can write

$$
\Delta f:=f(t, w(t))-f(t, v(t))=c(t) u(t) \quad \text { with }|c(t)| \leq m(t)
$$

and $c(t) \geq 0$ in case $f$ is increasing in $x$ (take $c(t)=\Delta f / u$ if $u \neq 0$ and $c(t)=0$ otherwise). In the general case $n>1$, the same is accomplished by writing $\Delta f_{i}$ as a sum of differences $\Delta_{1}, \ldots, \Delta_{n}$, where

$$
\Delta_{1}=f_{i}\left(t, w_{1}, v_{2}, \ldots, v_{n}\right)-f_{i}\left(t, v_{1}, v_{2}, \ldots, v_{n}\right), \ldots
$$

In this way one obtains

$$
f(t, w)-f(t, v)=C(t) u \quad \text { with }\left|c_{i j}(t)\right| \leq m(t)
$$

and $c_{i j}(t) \geq 0$ for $i \neq j$ because $f$ is quasimonotone increasing in $x$. Now the theorem follows from Theorem 1 .

4. Strong monotonicity. We are looking for conditions such that in Theorems 1 and 2 the set $\beta$ is empty, which means that in the conclusions 
strict inequality holds in $J_{0}$. The following notation is used. A measurable set $M \subset J$ is said to be dense at $a$ if the set $M \cap[a, a+\varepsilon]$ has positive measure for every $\varepsilon>0$. For measurable real-valued functions $\varphi, \psi$ we write $\varphi<\psi$ at $a+$ if the set $\{t \in J: \varphi(t)<\psi(t)\}$ is dense at $a$.

Theorem 3. Suppose $u(t)$ and $C(t)$ satisfy the assumptions of Theorem 1 , and there exists a nonempty index set $\alpha_{1}$ such that for $i \in \alpha_{1}$ either $u_{i}(a)>0$ or $u_{i}^{\prime}>(C u)_{i}$ at $a+$. If for every index set $\alpha_{0} \supset \alpha_{1}$ with $\beta_{0}=N \backslash \alpha_{0} \neq \emptyset$ there exist numbers $k \in \alpha_{0}, j \in \beta_{0}$ such that $c_{j k}>0$ at $a+$, then $u>0$ in $J_{0}$.

In particular, the assertion $u>0$ in $J_{0}$ holds under each of the following conditions:

(i) $u(a)>0$;

(ii) $u^{\prime}>C u$ at $a+$;

(iii) $u(a) \neq 0$ and the matrix $C(t)$ is irreducible at $a+$.

Irreducibility at $a+$ is defined as follows: For every nonempty index set $\alpha$ with $\beta=N \backslash \alpha \neq \emptyset$ there exist indices $k \in \alpha, j \in \beta$ such that $c_{j k}>0$ at $a+$.

Proof of Theorem 3. According to Theorem $1, u_{\alpha}>0$ in $J_{0}$ and $u_{\beta}=0$ in an interval $J_{\delta}=[a, a+\delta], \delta>0$. Assume $\beta \neq \emptyset$. Our assumptions imply that $\alpha \supset \alpha_{1}$. Putting $\alpha=\alpha_{0}$, we find indices $k \in \alpha, j \in \beta$ such that $c_{i j}>0$ at $a+$, which implies

$$
u_{j}^{\prime}=0 \geq \sum_{l} c_{j l} u_{l} \geq c_{j k} u_{k}>0 \quad \text { at } a+.
$$

This contradiction shows that $\beta=\emptyset$.

Now assume that the assumptions of Theorem 2 hold and that the set $\beta$ in the conclusion is not empty. We write $x=\left(x_{\alpha}, x_{\beta}\right), v(t)=\left(v_{\alpha}(t), v_{\beta}(t)\right), \ldots$ with an obvious meaning. Let $\delta=\min \left\{\delta_{j}: j \in \beta\right\}$. Then $v_{\beta}=w_{\beta}$ in $J_{\delta}=[a, a+\delta]$, and $P v \leq P w$ implies

$$
f_{\beta}\left(t, v_{\alpha}, v_{\beta}\right) \geq f_{\beta}\left(t, w_{\alpha}, w_{\beta}\right) .
$$

But from quasimonotonicity and $v \leq w$ we get $f_{\beta}\left(t, v_{\alpha}, v_{\beta}\right) \leq f_{\beta}\left(t, w_{\alpha}, v_{\beta}\right)$ and hence

$$
f_{\beta}\left(t, v_{\alpha}, v_{\beta}\right)=f_{\beta}\left(t, w_{\alpha}, v_{\beta}\right) \quad \text { and } \quad v_{\alpha}<w_{\alpha} \quad \text { in } J_{\delta}=(a, a+\delta],
$$

which implies, by the way, that $P_{\beta} v=P_{\beta} w$ in $J_{\delta}$. So, in order to obtain $\beta=\emptyset$, we must add an assumption which is incompatible with (5).

THEOREM 4. Suppose $v(t), w(t)$ and $f$ satisfy the assumptions of Theorem 2. Each of the following conditions is sufficient for the strong inequality $v<w$ in $J_{0}$ : 
(i) $v(a)<w(a)$;

(ii) $P v<P w$ at $a+$;

(iii) For every pair $(\alpha, \beta)$ of nonempty, disjoint index sets with $\alpha \cup \beta=N$ there are $j \in \beta, k \in \alpha$ such that $f_{j}(t, x)$ is strictly increasing in $x_{k}$ for $t \in M$, $x \in U$, where $M \subset J$ is dense at $a$ and $U$ is a neighborhood of $v(a)$.

Proof. It is obvious that $\beta$ is empty in cases (i), (ii). In case (iii) we use the notation $x=\left(x_{k}, \widetilde{x}_{k}\right)$ with $\widetilde{x}_{k} \in \mathbb{R}^{n-1}$. There is $\delta>0$ such that for $a<t \leq \delta$ and $0<s<\delta$,

$$
v(t) \in U, \quad v^{s}(t):=\left(v_{k}(t)+s, \widetilde{v}_{k}(t)\right) \in U
$$

and $v_{k}(t)+s \leq w_{k}(t)$, which implies $v^{s}(t) \leq w(t)$. It follows from quasimonotonicity of $f$ and the strict monotonicity of $f_{j}$ that

$$
f_{j}(t, v(t))<f_{j}\left(t, v^{s}(t)\right) \leq f_{j}(t, w(t)) \quad \text { for } t \in M .
$$

This is a contradiction to (5).

Remarks. 1 . If $f(t, x)$ is of class $C^{1}$ with respect to $x$, then (iii) follows from

(iii') The Jacobian $\partial f(t, x) / \partial x$ is irreducible for $t \in M, x \in U(v(a))$.

2. In Theorem 3 it was assumed that $f$ is defined in $J \times \mathbb{R}^{n}$. If $f$ is only defined in a set $G=J \times D$, where $D \subset \mathbb{R}^{n}$ is open, then it is naturally assumed that graph $v$, graph $w \subset G$. But in the representation of $\Delta f=$ $f(t, w(t))-f(t, v(t))$ as a sum of differences (cf. the proof of Theorem 2), the auxiliary points must also belong to $G$. This is the case if $D$ is an open $n$-dimensional interval. Yet convexity of $D$ suffices. The proof runs as follows. Let $x, y \in D, h=(y-x) / p$ and $x^{k}=x+k h(k=0, \ldots, p)$, in particular $x^{0}=x, x^{p}=y$. Then $f(t, y)-f(t, x)=\sum_{k=1}^{p}\left[f\left(t, x^{k}\right)-f\left(t, x^{k-1}\right)\right]$, and each of those differences can be treated as in the proof of Theorem 2 (the line segment $\overline{x y}$ has positive distance to the boundary of $D$, and for large $p$ all auxiliary points are close to $\overline{x y}$ ).

3. The two definitions of quasimonotonicity in Section 3 are not always equivalent if $f$ is only defined in $G=J \times D$. This fact was first observed by Ważewski [4] in 1950. He introduced two conditions (H) and (K): Condition $(\mathrm{H})$ is the one given above by means of inequalities, Condition $(\mathrm{K})$ requires that $f_{i}(t, x)$ is (weakly) increasing in $x_{j}$ for $i \neq j$. Obviously $(\mathrm{H})$ implies $(\mathrm{K})$, but $(\mathrm{K})$ implies $(\mathrm{H})$ only if the set $D$ has a certain property $P$ which is described in [4]. For example, convex sets have this property. What is really needed in inequalities is always the form $(\mathrm{H})$ first introduced by Ważewski. Let us remark that the general quasimonotonicity condition for differential equations in ordered Banach spaces (or topological vector spaces), which was given by Volkmann [2], is also of the $(\mathrm{H})$ type. 
4. We denote the solution of $y^{\prime}=f(t, y), y(a)=\eta$ by $y(t, \eta)$. Under the assumptions of Remark 1 the Jacobian $Z(t, \eta)=\partial y(t, \eta) / \partial \eta$ satisfies $Z_{i j}(t, \eta)>0$ for $t \geq a$ and all $i, j \in N$. This follows from Theorems 1 and 3 , since $Z(t, \eta)$ is a solution to the linear system

$$
Z^{\prime}=C(t) Z, \quad Z(0)=I, \quad \text { where } C(t)=\frac{\partial f}{\partial x}(t, y(t, \eta)) ;
$$

cf. [3], Theorem 13.X. The matrix $C(t)$ is essentially positive and irreducible. Note that the columns $z^{i}=\partial y(t, \eta) / \partial \eta_{i}$ satisfy $z_{i}^{\prime}=C(t) z_{i}, z_{i}(0) \neq 0$ and hence are positive in $J_{0}$. This is a simple proof of Theorem 1.1 in [1].

\section{References}

[1] M. Hirsch, Systems of differential equations that are competitive or cooperative, II: convergence almost everywhere, SIAM J. Math. Anal. 16 (1985), 423-439.

[2] P. Volkmann, Gewöhnliche Differentialgleichungen mit quasimonoton wachsenden Funktionen in topologischen Vektorräumen, Math. Z. 127 (1972), 157-164.

[3] W. Walter, Gewöhnliche Differentialgleichungen, 5th ed., Springer, 1993.

[4] T. Ważewski, Systèmes des équations et des inégalités différentielles ordinaires aux deuxièmes membres monotones et leurs applications, Ann. Soc. Polon. Math. 23 (1950), 112-166.

Mathematisches Institut I

Universität Karlsruhe

D-76128 Karlsruhe, Germany

E-mail: walter@ma1ws1.mathematik.uni-karlsruhe.de 